\title{
Making Progress through Molecular Attacks on Cancer
}

\author{
C.L. SAWYERS \\ Howard Hughes Medical Institute, Departments of Medicine, Medical and Molecular Pharmacology \\ and Urology, UCLA, Los Angeles, California 90095
}

\begin{abstract}
The success of kinase inhibitor therapy in chronic myeloid leukemia (CML) has validated the long-held thesis in the cancer research community that a precise molecular understanding of cancer can directly affect cancer therapy. Now that several years have passed since the approval of imatinib/Gleevec for CML treatment, we have a greater appreciation for the challenges involved in effectively deploying these agents in the clinic. In this paper, I review recent events in the treatment of CML and highlight early applications of kinase inhibitor therapy to other diseases such as glioblastoma. I conclude with a vision that it may be possible, through analysis of tumor proteins secreted into serum, to track distinct molecular features of various cancers in order to select appropriate molecularly targeted therapy and measure treatment response. This new science of cancer biomarkers could radically transform the conduct of clinical trials and speed the evaluation of new molecularly targeted agents.
\end{abstract}

\section{TUMORS WITH KINASE MUTATIONS ARE KINASE-DEPENDENT AND RESPOND TO KINASE INHIBITORS}

A central theme that has emerged from this work is the notion of "kinase-dependent" cancer; i.e., those cancers whose growth is driven by a specific kinase or set of kinases (Sawyers 2003). By definition, such cancers should respond (shrink) when exposed to an inhibitor that effectively blocks the enzymatic activity of the responsible kinase. Although CML serves as a paradigm, it is unique in that essentially all patients with clinical CML share a common molecular abnormality, activation of the ABL tyrosine kinase by chromosome translocation. It is perhaps more instructive to consider other diseases where only subsets of patients respond to these drugs. Examples include gastrointestinal stromal tumors (GIST), chronic myelomonocytic leukemia (CMML), hypereosinophilic syndrome (HES), and bronchoalveolar lung cancer. In each of these cases, clinical sensitivity (defined as objective response or shrinkage, measured radiographically) to a kinase inhibitor has been correlated with a mutation in the gene encoding the target kinase that alters its biological potency, creating an oncogenic allele that drives the growth of that cancer. Table 1 (top) summarizes the mutant kinase target and effective kinase inhibitor for each of these tumors.
Although lung cancer patients with EGFR kinase domain mutations clearly have a greater chance of objective response when treated with inhibitors such as Iressa or Tarceva, there is much debate in the lung cancer community about whether these mutations predict for any longterm clinical benefit to patients, since there appears to be no improvement in survival. One possibility is that drug resistance (and relapse) develops in responders before a survival benefit is realized, in which case a second drug that combats or prevents resistance is needed. In CML, there is strong precedent in support of this view, because the mechanism of resistance to imatinib has been defined and novel ABL kinase inhibitors have been shown to work (Gorre et al. 2001; Shah et al. 2002) (see below). Initial data from several groups, as presented by Dr. Varmus (Varmus et al., this volume) and Dr. Haber (Haber et al., this volume), suggest that the same may be true in lung cancer, since resistance mechanisms are similar and novel inhibitors with efficacy against resistant cells have been described. The long-term data in lung cancer patients treated with EGFR inhibitors are further complicated by the evidence for a small but significant survival benefit to treated patients without known EGFR mutations. Although these data seem paradoxical at present, further molecular characterization of these patients for other mutations or pathway abnormalities is likely to clarify the situation, based on precedent recently established

Table 1. Kinase-driven Cancers with Kinase Mutations

\begin{tabular}{llll}
\hline Disease & Kinase & Mechanism & Drug \\
\hline CML & ABL & fusion protein & Gleevec \\
GI stromal tumor & KIT & mutation & Gleevec \\
Hypereosinophilic syndrome & PDGFR $\alpha$ & fusion protein & Gleevec \\
CMML & PDGsion protein & Gleevec \\
Lung cancer & EGFR & mutation & Iressa/Tarceva \\
AML & Flt3 & mutation & $?$ \\
Melanoma & B-Raf & mutation & $?$ \\
Lung cancer & Her2 & mutation & $?$ \\
Breast cancer, etc. & PI3K $(\mathrm{p} 110 \alpha)$ & mutation & $?$ \\
Polycythemia vera, ET & JAK2 & mutation & $?$ \\
\hline
\end{tabular}


in glioblastoma (Mellinghoff et al. 2005) (see below).

Assuming the paradigm that mutant kinases predict for kinase dependence continues to hold up, there is great optimism that kinase inhibitors will be effective in other diseases with known kinase mutations. Currently, the list of candidates is not very long (B-Raf in melanoma, Her2 in lung cancer, Flt3 in AML, JAK2 in myeloproliferative disease) (Table 1, bottom), but there is optimism that it will continue to grow with time. Specifically, human cancer kinome resequencing efforts are currently under way and have identified novel kinase mutations in subsets of patients. Although the actual frequency of specific mutations in certain cancers is likely to be low, there is great interest in developing a comprehensive catalog of all kinase (and other gene) mutations found in human cancers. Although the magnitude and cost of such a project are under debate, there is little doubt that discovery of additional kinase mutations could rapidly have clinical impact. Small-molecule inhibitors to mutant kinases can now be readily identified through standard screening approaches, followed by clinical evaluation in patients most likely to respond; i.e., those whose tumors contain mutations in the target kinase.

\section{IMPORTANCE OF TUMOR SUPPRESSOR GENE STATUS IN RESPONSE TO KINASE INHIBITORS}

It is important to recognize certain complexities that may influence tumor response to kinase inhibitors, even in tumors with a mutation in the target kinase. Analysis of glioblastoma patients treated with EGFR inhibitors by the UCLA Neurooncology Group (Ingo Mellinghoff, Tim Cloughesy, Paul Mischel) serves as an example. The response rate to the drugs (Iressa or Tarceva) in relapsed glioma is $\sim 15 \%$ in phase II studies. Of 49 such patients treated at UCLA, 7 had dramatic, radiographically documented objective responses. Molecular profiling of the EGFR in all these patients revealed that 6 of these 7 patients had mutations in the extracellular domain (called EGFR variant iii). (Of note, no patients had mutations in the EGFR kinase domain, thereby distinguishing this mechanism from that found in lung cancer.) However, 6 of 19 patients who rapidly progressed through EGFR inhibitor therapy also had EGFRviii mutations. The variable that distinguishes these two groups is presence or absence of the PTEN tumor suppressor gene, which was expressed in wild-type form in all of the responding patients but in only 2 of the progressive disease patients with EGFRviii mutations. These data were confirmed in an independent sample set and suggest that molecular assessment of both EGFRviii and PTEN is required to define sensitivity to EGFR inhibitors in glioblastoma patients (Mellinghoff et al. 2005). The broader implication is that future application of patient-tailored kinase inhibitor therapy is likely to require evaluation of a suite of molecular variables for optimal predictive power.

Loss of the PTEN tumor suppressor gene is also of interest for potential therapeutic opportunities because PTEN is a lipid phosphatase that regulates the PI3-kinase pathway (Vivanco and Sawyers 2002). Tumors lacking PTEN have increased activation of PI3K and downstream effectors such as Akt and mTOR, raising the possibility of dependence on these kinases in the absence of direct mutation of the kinase. We and other workers have demonstrated a synthetic lethal-like relationship between PTEN loss and mTOR in various mouse models treated with the mTOR inhibitor rapamycin, which causes cytostasis or regression of PTEN null tumors (Neshat et al. 2001; Podsypanina et al. 2001; Majumder et al. 2004; Wendel et al. 2004). Based on this work, clinical trials of mTOR inhibitors have been initiated with the goal of assessing response rate in patients with PTEN mutant cancers.

At UCLA we have addressed this question in glioblastoma and in prostate cancer, based on the relatively high frequency of PTEN loss $(\sim 40 \%)$ in these two diseases. A critical first step in both trials was to establish clinically robust assays of PTEN pathway status to select appropriate patients for these clinical trials. Using immunohistochemistry, we demonstrated that PTEN expression and downstream pathway activation could be measured in formalin-fixed, paraffin-embedded prostate and glioma samples (Choe et al. 2003; Thomas et al. 2004). The second step was to design trials so that PTEN pathway status could be determined from biopsy material and pathway inhibition could be measured using appropriate pharmacodynamic readouts. For these reasons, we used a neoadjuvant clinical design where patients are treated with mTOR inhibitor briefly (1-4 weeks) prior to a planned surgical resection, allowing us to measure pathway status in resected tumor. In both the prostate and the glioma trials, we were successful in defining doses of mTOR inhibitor that inhibit target in tumor cells with minimal side effects. Anti-tumor activity is suggested by changes in proliferation index, but formal efficacy data will require phase II studies.

\section{ACQUIRED RESISTANCE TO KINASE INHIBITORS: MECHANISMS AND POTENTIAL SOLUTIONS}

A second issue complicating the success of kinase inhibitor therapy is acquired resistance, defined as disease relapse on continuous therapy after an initial response. First recognized as a significant problem initially in advanced-stage CML patients, acquired resistance also occurs in chronic-phase CML, GIST, HES, and bronchoalveolar lung cancer. The best understanding of resistance mechanisms comes from CML, where $>85 \%$ of relapsed patients have mutations in the ABL kinase domain that alter drug sensitivity (Shah et al. 2002). Of note, similar mechanisms have been reported for GIST and HES but explored in less detail.

In the case of $\mathrm{ABL}, 38$ different mutations have been reported to date, but 3-4 mutations account for $60-70 \%$ of all cases. Insights into how these mutations cause resistance became apparent through the solution by John Kuriyan's group of the co-crystal structure of imatinib bound to ABL (Schindler et al. 2000). Curiously, only a small number of the mutations occur at contact residues 
where substitution of the new amino acid leads to loss of a hydrogen-bond donor or steric hindrance due to bulkier side chains. Rather, the majority of mutations are at residues that appear, based on structural modeling studies, to alter the conformational flexibility of ABL such that it can no longer achieve the closed, inactive conformation required for optimal imatinib binding (Shah et al. 2002).

These structural studies raise the possibility that a second ABL kinase inhibitor which binds in a less conformation-dependent fashion may have activity against certain imatinib-resistant mutants. We tested this hypothesis using dual SRC/ABL kinase inhibitors that can bind ABL in the active or inactive conformation. One such compound, BMS-354825 (also called dasatinib), blocked the growth of murine hematopoietic cells transformed by all but one imatinib-resistant BCR-ABL mutant in culture and showed anti-leukemic activity in mouse models (Shah et al. 2004). This compound also induced hematologic and cytogenetic remissions in a high fraction of imatinib-resistant CML patients in a phase I trial with minimal side effects (Sawyers et al. 2004, 2005). Phase II studies are currently under way with promising data presented recently with 6 months followup (Guilhot et al. 2005; Hochhaus et al. 2005; Ottmann et al. 2005; Talpaz et al. 2005).

We have also examined potential mechanisms of resistance to BMS-345825 by saturation mutagenesis. Unlike imatinib, resistance occurs almost exclusively through mutations at drug contact residues, presumably due to less conformation-stringent binding requirements. In addition, some mutations were isolated that confer resistance to BMS-354825 but not to imatinib (Burgess et al. 2005). These data provide evidence in favor of either sequential or combination therapy with these two compounds for CML.

\section{NEXT STEPS IN CLINICAL TRIAL DESIGN: THE NEED FOR ACCESSIBLE BIOMARKERS}

The neoadjuvant trial designs discussed above for testing mTOR inhibitors in PTEN-null cancers provide a unique opportunity to obtain tumor tissue to make molecular measurements of pathway status, but the requirement for tissue acquisition places significant limitations on patient eligibility. Noninvasive technologies for measuring pathway activation would greatly transform the conduct of clinical trials. Over the past several years, there has been a growing interest in defining serum biomarkers for cancer diagnosis and early detection, based on evidence of distinct patterns of certain serum proteins detected in cancer patients using proteomic technologies. Our group is examining the possibility that distinct sets of proteins secreted by tumor cells with different genetic pathway mutations might be used to measure pathway status in serum. As a first step, we have conducted a whole mouse transcriptome profiling experiment of two genetically engineered mouse prostate cancer models designed to mimic lesions found in human prostate cancer, one with transgenic expression of c-Myc and the other with conditional deletion of PTEN (Ellwood-Yen et al. 2003; Wang et al. 2003). In preliminary studies conducted with the groups of Hong Wu and Tom Graeber at UCLA, we have defined gene expression signatures that easily distinguish PTEN and Myc-driven cancers, even when the analysis is restricted to genes predicted to encode only secreted proteins. If these predicted proteins are indeed secreted and measurable in serum, one can envision using a suite of serum biomarkers to define the genetic status of a patient's tumor and determine pathway inhibition in response to therapy. Such tools, coupled with advances in probe development for molecular imaging, could transform clinical cancer diagnosis and rapidly allow therapies to be appropriately tailored to the genetic makeup of the tumor. These technological advances are critical for successful application of molecularly targeted therapies on a larger scale against a range of malignancies beyond chronic myeloid leukemia.

\section{ACKNOWLEDGMENTS}

I thank many colleagues at UCLA who have contributed to this studies, including Neil Shah, Ingo Mellinghoff, Tim Cloughesy, Paul Mischel, Hong Wu, Tom Graeber, Ron Paquette, Michael Burgess, and John Nicoll. Work in my laboratory is supported by the Howard Hughes Medical Institute, the Leukemia and Lymphoma Society, the National Cancer Institute, and the Doris Duke Charitable Foundation.

\section{REFERENCES}

Burgess M.R., Skaggs B.J., Shah N.P., Lee F.Y., and Sawyers C.L. 2005. Comparative analysis of two clinically active BCRABL kinase inhibitors reveals the role of conformation-specific binding in resistance. Proc. Natl. Acad. Sci. 102: 3395.

Choe G., Horvath S., Cloughesy T.F., Crosby K., Seligson D., Palotie A., Inge L., Smith B.L., Sawyers C.L., and Mischel P.S. 2003. Analysis of the phosphatidylinositol 3'-kinase signaling pathway in glioblastoma patients in vivo. Cancer Res. 63: 2742 .

Ellwood-Yen K., Graeber T.G., Wongvipat J., Iruela-Arispe M.L., Zhang J., Matusik R., Thomas G.V., and Sawyers C.L. 2003. Myc-driven murine prostate cancer shares molecular features with human prostate tumors. Cancer Cell 4: 223.

Gorre M.E., Mohammed M., Ellwood K., Hsu N., Paquette R., Rao P.N., and Sawyers C.L. 2001. Clinical resistance to STI571 cancer therapy caused by BCR-ABL gene mutation or amplification. Science 293: 876.

Guilhot F., Apperley J.F., Shah N., Kim D.W., Grigg A., Cheng S., Iyer M., and Cortes J. 2005. A phase II study of Dasatinib in patients with accelerated phase chronic myeloid leukemia (CML) who are resistant or intolerant to Imatinib: First results of the CA180005 'START-A' study. American Society of Hematology 47th Annual Meeting, Abstract 39.

Hochhaus A., Baccarani M., Sawyers C., Nagler A., Facon T., Goldberg S.L., Cervantes F., Larson R.A., Voi M., Ezzeddine R., and Kantarjian H. 2005. Efficacy of Dasatinib in patients with chronic phase Philadelphia chromosome-positive CML resistant or intolerant to Imatinib: First results of the CA180013 'START-C' phase II study. American Society of Hematology 47th Annual Meeting, Abstract 41.

Majumder P.K., Febbo P.G., Bikoff R., Berger R., Xue Q., McMahon L.M., Manola J., Brugarolas J., McDonnell T.J., Golub T.R., Loda M., Lane H.A., and Sellers W.R. 2004. mTOR inhibition reverses Akt-dependent prostate intraepithelial neoplasia through regulation of apoptotic and HIF-1dependent pathways. Nat. Med. 10: 594. 
Mellinghoff I.K., Wang M.Y., Vivanco I., Haas-Kogan D.A., Zhu S., Dia E.Q., Lu K.V., Yoshimoto K., Huang J.H., Chute D.J., Riggs B.L., Horvath S., Liau L.M., Cavenee W.K., Rao P.N., Beroukhim R., Peck T.C., Lee J.C., Sellers W.R., Stokoe D., Prados M., Cloughesy T.F., Sawyers C.L., and Mischel P. 2005. Molecular determinants of the response of glioblastomas to EGFR kinase inhibitors. N. Engl. J. Med. 353: 2012.

Neshat M.S., Mellinghoff I.K., Tran C., Stiles B., Thomas G., Petersen R., Frost P., Gibbons J.J., Wu H., and Sawyers C.L. 2001. Enhanced sensitivity of PTEN deficient tumors to inhibition of FRAP/mTOR. Proc. Natl. Acad. Sci. 98: 10314.

Ottmann O.G., Martinelli G., Dombret H., Kantarjian H., Hochhaus A., Simonsson B., Aloe A., Apanovitch A., and Shah N. 2005. A phase II study of Dasatinib in patients with chronic myeloid leukemia (CML) in lymphoid blast crisis or Philadelphia-chromosome positive acute lymphoblastic leukemia (Ph+ ALL) who are resistant or intolerant to Imatinib: The 'START-L' CA180015 study. American Society of Hematology 47th Annual Meeting, Abstract 42.

Podsypanina K., Lee R.T., Politis C., Hennessy I., Crane A., Puc J., Neshat M., Wang H., Yang L., Gibbons J., Frost P., Dreisbach V., Blenis J., Gaciong Z., Fisher P., Sawyers C., Hedrick-Ellenson L., and Parsons R. 2001. An inhibitor of mTOR reduces neoplasia and normalizes p70/S6 kinase activity in Pten+/-mice. Proc. Natl. Acad. Sci. 98: 10320.

Sawyers C.L. 2003. Opportunities and challenges in the development of kinase inhibitor therapy for cancer. Genes Dev. 17: 2998.

Sawyers C.L., Kantarjian H., Shah N., Cortes J., Paquette R., Donato N., Nicoll J., Bleickardt E., Chen T.T., and Talpaz M. 2005. Dasatinib (BMS-354825) in patients with chronic myeloid leukemia (CML) and Philadelphia-chromosome positive acute lymphoblastic leukemia (Ph+ ALL) who are resistant or intolerant to Imatinib: Update of a phase I study. American Society of Hematology 47th Annual Meeting, Abstract 38.

Sawyers C.L., Shah N.P., Kantarjian H.M., Donato N., Nicoll J., Bai S.A., Huang F., Clark E., DeCillis A.P., and Talpaz M. 2004. Hematologic and cytogenetic responses in Imatinib-re- sistant chronic phase chronic myeloid leukemia patients treated with the dual SRC/ABL kinase inhibitor BMS354825: Results from a phase I dose escalation study. American Society of Hematology 46th Annual Meeting, Abstract 1. Schindler T., Bornmann W., Pellicena P., Miller W.T., Clarkson B., and Kuriyan J. 2000. Structural mechanism for STI-571 inhibition of abelson tyrosine kinase. Science 289: 1938.

Shah N.P., Tran C., Lee F.Y., Chen P., Norris D., and Sawyers C.L. 2004. Overriding Imatinib resistance with a novel ABL kinase inhibitor. Science 305: 399.

Shah N.P., Nicoll J.M., Nagar B., Gorre M.E., Paquette R.L., Kuriyan J., and Sawyers C.L. 2002. Multiple BCR-ABL kinase domain mutations confer polyclonal resistance to the tyrosine kinase inhibitor Imatinib (STI571) in chronic phase and blast crisis chronic myeloid leukemia. Cancer Cell 2: 117.

Talpaz M., Rousselot P., Kim D.W., Guilhot F., Corm S., Bleickardt E., Zink R., Rosti G., Coutre S., and Sawyers C. 2005. A phase II study of Dasatinib in patients with chronic myeloid leukemia (CML) in myeloid blast crisis who are resistant or intolerant to Imatinib: First results of the CA180006 'START-B' study. American Society of Hematology 47th Annual Meeting, Abstract 40.

Thomas G.V., Horvath S., Smith B.L., Crosby K., Lebel L.A., Schrage M., Said J., De Kernion J., Reiter R.E., and Sawyers C.L. 2004. Antibody-based profiling of the phosphoinositide 3-kinase pathway in clinical prostate cancer. Clin. Cancer Res. 10: 8351.

Vivanco I. and Sawyers C.L. 2002. The phosphatidylinositol 3kinase AKT pathway in human cancer. Nat. Rev. Cancer 2: 489.

Wang S., Gao J., Lei Q., Rozengurt N., Pritchard C., Jiao J., Thomas G.V., Li G., Roy-Burman P., Nelson P.S., Liu X., and Wu H. 2003. Prostate-specific deletion of the murine Pten tumor suppressor gene leads to metastatic prostate cancer. Cancer Cell 4: 209.

Wendel H.G., De Stanchina E., Fridman J.S., Malina A., Ray S., Kogan S., Cordon-Cardo C., Pelletier J., and Lowe S.W. 2004. Survival signalling by Akt and eIF4E in oncogenesis and cancer therapy. Nature 428: 332. 


\section{$8_{8}^{\infty} \mathrm{CSH} \&$ Cold Spring Harbor Symposia SYMPOSIA on Quantitative Biology}

\section{Making Progress through Molecular Attacks on Cancer}

\section{C.L. SAWYERS}

Cold Spring Harb Symp Quant Biol 2005 70: 479-482

Access the most recent version at doi:10.1101/sqb.2005.70.034

References This article cites 16 articles, 9 of which can be accessed free at:

http://symposium.cshlp.org/content/70/479.full.html\#ref-list-1

\section{License}

Email Alerting Receive free email alerts when new articles cite this article - sign up in Service the box at the top right corner of the article or click here. 